\title{
Lingual Lymphangioma Ablation With High Power Diode Laser: A Case Report
}

\author{
Amanda Vieira Aires ${ }^{1}$, Camila de Nazaré Alves de Oliveira Kato ${ }^{1 *}$, Leni Verônica de Oliveira Silva ${ }^{1}$, Rodrigo \\ Soares de Andrade², Hercílio Martelli Júnior ${ }^{3}$, Marcus Vinicius Lucas Ferreira ${ }^{1}$, Ricardo Alves Mesquita ${ }^{1}$
}

${ }^{1}$ School of Dentistry, Universidade Federal de Minas Gerais, Belo Horizonte, Minas Gerais, Brazil

${ }^{2}$ School of Dentistry of Piracicaba, Universidade Estadual de Campinas, Piracicaba, São Paulo, Brazil

${ }^{3}$ State University of Montes Claros (Unimontes), Montes Claros, MG 39401-089, Brazil

\author{
*Correspondence to \\ Camila de Nazaré Alves de \\ Oliveira Kato, DDS, PhD student; \\ Universidade Federal de \\ Minas Gerais, Faculdade de \\ Odontologia, sala 3202D. \\ Av. Antônio Carlos, 6667 \\ Pampulha 31.270-010, Belo \\ Horizonte, MG, Brasil. \\ Tel: 55-31-31-3409-2499 \\ Email: cnao20@yahoo.com.br
}

Published online March 15, 2020

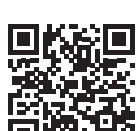
\section{然}

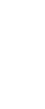

\section{Introduction}

Lymphangioma or lymphatic malformation is a benign and congenital disorder that arises from lymphatics with a deficient connection of drainage to other lymphatic vessels or veins..$^{1-4}$ For microcystic lymphangioma, clinical and complementary imaging exams, such as ultrasonography (US), are recommended as diagnostic tools. Histopathological examination is performed only in microcystic lesions with atypical clinical profiles. ${ }^{2}$ Several types of treatment have been described in accordance with the classification and location of the lymphangioma. Surgical excision is the treatment most commonly performed, but it has limitations since microcystic lesions have the capacity to infiltrate into adjacent tissues and the complete removal of the lesion is difficult. In addition, there are high rates of recurrence and morbidity associated with this form of treatment. ${ }^{1,3-6}$ Sclerotherapy is an alternative therapy, but studies reported the lack of standardization of the dosage, application intervals, and adverse effects, with better results observed in macrocystic lesions than in microcystic or circumscribed lesions. ${ }^{5-7}$ Carbon dioxide $\left(\mathrm{CO}_{2}\right)$ and the Nd:YAG laser have previously been successfully used to treat microcystic lymphangioma. ${ }^{3-5}$ Despite the reports of postoperative pain and edema after the ablation of lesions, recurrence was uncommon. ${ }^{4}$ The high cost of $\mathrm{CO}_{2}$ is an added disadvantage. ${ }^{8}$ Due to the good results obtained by the use of lasers in the treatment of vascular anomalies, mainly venous malformations, ${ }^{3,4}$ we describe the diode laser ablation of the microcystic lymphangioma on the dorsum of the tongue.

\section{Case Report}

The patient, a 9-year-old black boy, accompanied by his mother was referred to the oral pathology service. The complaint was pain, bleeding, and discomfort during chewing. The patient's mother reported the presence of the lesion from birth with periods of growth and decline. During the intraoral examination, multiple vesicles of different sizes were observed, varying from white to transparent and erythematous, with smooth surfaces and poorly defined borders, affecting an area of approximately $3 \mathrm{~cm}^{2}$ (Figure 1a). The complete blood count and the US exam were requested. Laboratory tests showed no changes and US showed low-flow vascular malformation in the tongue (Figure 1b). Clinical and imaging data led to the diagnosis of microcystic lymphangioma.

The patient and his mother were instructed to carry out careful oral hygiene, especially in the region of the lingual dorsum, with gauze moistened with chlorhexidine gluconate $0.12 \%$, alcohol-free solution $\left(\right.$ Periogard $^{\circledR}$, 


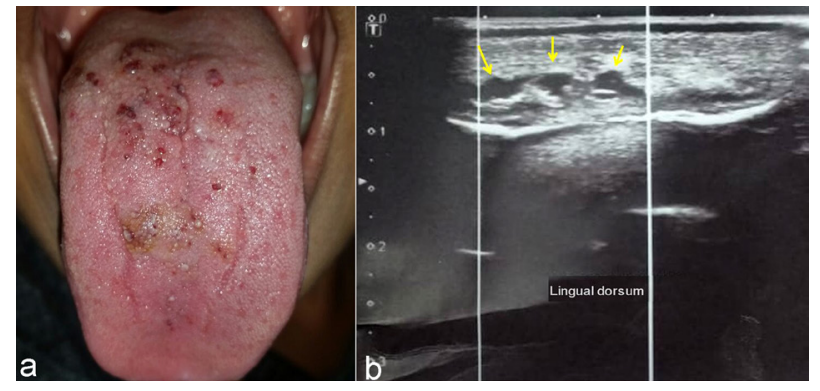

Figure 1. (a) Initial appearance of the lesion; (b) Initial ultrasound image presented low-flow vascular malformation (yellow arrows).

Colgate-Palmolive, São Paulo-SP, Brazil). After 20 days of correct cleaning of the lesioned area, there was a significant improvement and reduction in the microcystic lymphangioma (Figure 2), without signs of inflammation. At this time, the proposed treatment was ablation with a Gallium-Indium-Arsenide diode Laser (THERA LASE, DMC Equipment's, São Carlos, SP, Brazil) with an $808 \mathrm{~nm}$ wavelength, $4 \mathrm{~W}$ continuous output power, and an optical fiber $400 \mu \mathrm{m}$ in diameter with an area of $0.001 \mathrm{~cm}^{2}$. For the procedures, power between $2.0 \mathrm{~W}$ and $2.5 \mathrm{~W}$ was used, resulting in an irradiance ranging between $2000 \mathrm{~W} /$ $\mathrm{cm}^{2}$ and $2500 \mathrm{~W} / \mathrm{cm}^{2}$.

Two sessions with an interval of 3 months between them were necessary to treat the lesion (Figure 3 ). The procedures were performed under local anesthesia (lidocaine 2\% and epinephrine 1:100000). In the first session, the total area of $0.75 \mathrm{~cm}^{2}$ was treated (Figure $3 \mathrm{a}-3 \mathrm{~b})$. The diode laser parameters used were $2000 \mathrm{~mW}$ power and $1006 \mathrm{~J}$ total energy, and the laser was turned on for 8 minutes and 23 seconds. In the second session, the lesion covered an area of $1.2 \mathrm{~cm}^{2}$ (Figure $3 \mathrm{e}-3 \mathrm{f}$ ), and the laser parameters used were $2500 \mathrm{~mW}$ power and 540 $\mathrm{J}$ total energy, and the laser was turned on for 3 minutes and 36 seconds. In the second session, the irradiated area was bigger than it was in the first because of the decision to irradiate other spots that were not treated in the first session. The laser operator decided to use an additional $500 \mathrm{~mW}$ of power in the second session because no significant thermal harm resulting from the first session

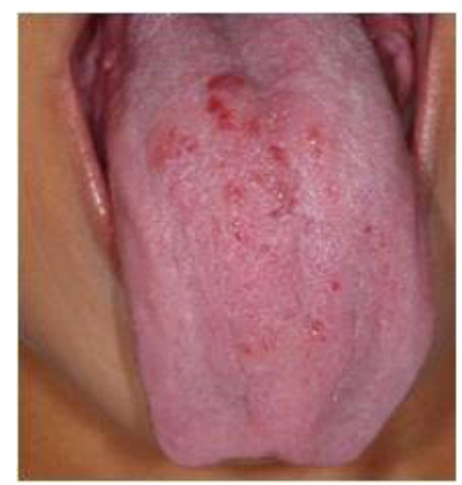

Figure 2. Clinical appearance of the lesion after 20 days of hygiene. was shown in the postoperative period. In the second session, a lower amount of total energy was used than that used in the first session because the thickness of the lesion had been reduced.

Two days after the first ablation session, the patient reported edema and difficulty in chewing. The hygiene instructions had not been followed correctly and there was a secondary infection of the tongue due to poor oral hygiene. The patient and his mother were again instructed about the hygiene of the tongue. After 7 days, there was remission of the infection, with a reduction in edema and discomfort, and the surgical wound was healing well (Figure $3 \mathrm{c}$ and $3 \mathrm{~d}$ ). As this was a rare lesion and laser application was usually performed at the clinic with the purpose of avoiding discomfort and the manifestation of other infections, we recommended prior antibiotic therapy (amoxicillin $250 \mathrm{mg} / 5 \mathrm{~mL}-1 \mathrm{~mL} / \mathrm{kg} / \mathrm{d}$ - suspension), initiated one day before the second laser session.

Clinical follow-up was performed for 3, 6 and 12 months. Until the last follow-up there were no signs of recurrence of the lesion or any other symptom reported by the patient (Figure $4 \mathrm{a}$ ). US exam in the region of the lesion after one year (Figure $4 \mathrm{~b}$ ) showed fibrotic tissue instead of microcystic lymphangioma, which demonstrated the effectiveness and safety of the diode laser treatment.

\section{Discussion}

Lymphangioma is usually classified according to clinical and histopathological characteristics. The diameter of the lesions allows their classification into three types: microcystic or circumscribed, macrocystic, and mixed., ${ }^{2,9}$ Histologically, lymphangioma is differentiated into

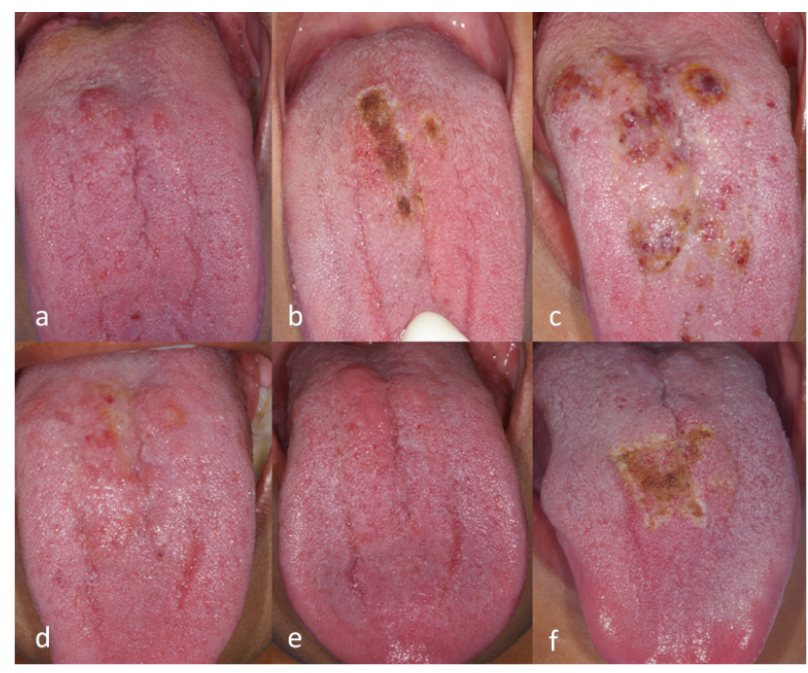

Figure 3. (a) Preoperative images of the first ablation session; (b) Dorsum of the tongue in the immediate postoperative period after the first ablation session with the diode laser; (C) Return after two days of treatment. The presence of secondary infection and edema was observed in the dorsum of the tongue; (d) After 7 days of performing oral hygiene correctly; (e) Preoperative images of the second ablation session; (f) Immediate postoperative images after the second ablation session. 


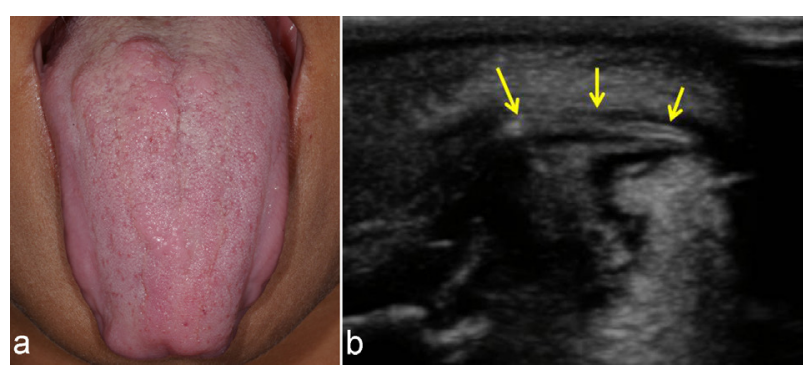

Figure 4. (a) Follow-up of the patient after 12 months; (b) Ultrasound image captured 12 months after the treatment.

capillary, cavernous, and cystic (cystic hygroma) types according to vessel size. ${ }^{2}$ US exam, a low-cost, rapid and non-ionizing radiation test, is considered a satisfactory resource to aid in the diagnosis of lymphangioma, ${ }^{1-5}$ especially for reporting the content, location, and extent of the lesion. The main reasons for therapeutic intervention in lymphangioma are functional, such as chewing, speech, and esthetic impairment. ${ }^{6,7}$ In addition, intermittent swelling may occur due to trauma or secondary infections. ${ }^{3}$

The treatment options for oral lymphangioma vary according to the size, location, and extent of the lesion. There is no standardized treatment in the literature and the treatment chosen according to the experience of the team responsible for the case considering the individuality of the patient. ${ }^{1}$ Surgical excision is the most common form of treatment, but nevertheless high rates of recurrence and possible complications such as bleeding, infections, and deformities have been observed. ${ }^{1,4,7}$ Sclerosing substances are frequently used, with bleomycin and OK-432 (Picibanil) being the most common. However, some authors have suggested that sclerotherapy is more effective in the treatment of macrocystic lymphangioma. ${ }^{1,6}$ The CO2, Nd:YAG and diode lasers have previously been successfully used in the treatment of microcystic lymphangioma..$^{3-5}$ The diode laser has some advantages when compared with $\mathrm{CO} 2$ and Nd:YAG lasers, related to cost (low) and equipment size (smaller). They have different wavelengths, but all of them are used for small soft tissue surgeries in the oral cavity.

The active medium of the diode laser is a solid-state semiconductor made of aluminum, gallium, arsenide, and occasionally indium. This active medium produces wavelengths from 810 to $980 \mathrm{~nm}$ in the infrared range and is highly efficient, thus reducing energy expenditure of laser operation. ${ }^{9}$ It has the ability to remove or cauterize abnormal vessels. ${ }^{10,11}$ In order to avoid a thermal effect, the operator performed continuous movements without stopping the optical fiber at a single point of the lesion. In addition, in the present case, the diode laser was used in the continuous mode, which the beam of laser light is emitted without interruption. The advantage of this mode is faster tissue removal, but there is greater potential to accumulate heat and cause collateral damage to adjacent tissues. ${ }^{11}$ Furthermore, laser treatment induces tissue fibrosis at the site of the lesion, ${ }^{4}$ and the patient should be informed about fibrous scar esthetics at the end of treatment.

An $808 \mathrm{~nm}$ diode laser has high absorption in hemoglobin and low absorption in bones and teeth. This interaction of the laser with the tissue allows the phenomena of cauterization, ablation, and carbonization that occur according to the temperature in the target area. We chose to ablate the lesion due to the advantages that the laser offers. When the surgical site reaches a temperature of $100^{\circ} \mathrm{C}$, ablation occurs. The postoperative healing process is advantageous since the laser acts with the increase in temperature so that small blood and lymphatic vessels are dehydrated and collated, leading to hemostasis and reduction in postoperative edema. Disinfection of the target tissue due to high temperature was also observed, and in most cases, there was no need for suturing. ${ }^{10,11}$

The team chose to prescribe preventive antibiotic therapy due to secondary infection after the first laser session, poor hygiene practiced by the patient, low socioeconomic status of the family, localization of the lesion in the tongue, where there is a high number of bacteria, and difficulty of performing oral hygiene in the postoperative period. The use of antibiotics in laser surgeries is mentioned in the literature, in patients who did not constitute the risk group, although there was no indication, ${ }^{12}$ and in the evaluation after surgery, they presented pain and edema but no serious complication such as secondary infection in the operated area. ${ }^{4}$

\section{Conclusion}

The diode laser use reduced the intraoperative time; in addition, minimal bleeding was observed and there was no need for suturing. Postoperative pain and edema were also minimal. Therefore, in this case of lymphangioma, the diode laser was an effective and safe therapeutic resource since it allowed patient comfort, ease of use, and efficiency in the dental surgeon's work, thus suggesting that it can be explored as an option to treat these abnormalities in the mouth.

\section{Ethical Considerations}

Informed consent was obtained from the patient and his mother for the publication of this report.

\section{Conflict of Interests}

The authors declare no conflict of interest.

\section{Acknowledgment}

This work was supported by the Conselho Nacional de Desenvolvimento Científico e Tecnológico - CNPq [Grant No. CNPq \#305493/2018-3 and \#455644/2018-1].

\section{References}

1. Elluru RG, Balakrishnan K, Padua HM. Lymphatic 
malformations: diagnosis and management. Semin Pediatr Surg. 2014;23(4):178-185. doi: 10.1053/j. sempedsurg.2014.07.002.

2. Ganesh C, Sangeetha GS, Narayanan V, Umamaheswari TN. Lymphangioma circumscriptum in an adult: an unusual oral presentation. J Clin Imaging Sci. 2013;3:44. doi: 10.4103/2156-7514.120779.

3. Zhou Q, Zheng JW, Mai HM, Luo QF, Fan XD, Su LX, et al. Treatment guidelines of lymphatic malformations of the head and neck. Oral Oncol. 2011;47(12):1105-9. doi: 10.1016/j.oraloncology.2011.08.001.

4. Nammour S, Vanheusden A, Namour A, Zeinoun T. Evaluation of a new method for the treatment of invasive, diffuse, and unexcisable lymphangiomas of the oral cavity with defocus $\mathrm{CO}_{2}$ laser beam: a 20-year followup. Photomed Laser Surg. 2016;34(2):82-7. doi: 10.1089/ pho.2015.4019.

5. Alghonaim Y, Varshney R, Sands N, Daniel SJ. Coblation technique as an alternative treatment modality for oral lymphangioma. Int $J$ Pediatr Otorhinolaryngol. 2012;76(10):1526-7. doi: 10.1016/j.ijporl.2012.06.014.

6. Churchill P, Otal D, Pemberton J, Ali A, Flageole H, Walton JM. Sclerotherapy for lymphatic malformations in children: a scoping review. J Pediatr Surg. 2011;46(5):912-22. doi: 10.1016/j.jpedsurg.2011.02.027.

7. Kataria P, Passey JC, Agarwal AK. Lymphangioma circumscriptum of the tongue: successful treatment using intralesional bleomycin. $J$ Laryngol Otol. 2009;123(12):1390-2. doi: 10.1017/S0022215109005714.

8. Verma SK, Maheshwari S, Singh RK, Chaudhari PK. Laser in dentistry: an innovative tool in modern dental practice. Natl J Maxillofac Surg. 2012;3(2):124-32. doi: 10.4103/09755950.111342 .

9. International Society for the Study of Vascular Anomalies. ISSVA Classification of Vascular Anomalies. 15] Available from: https://www.issva.org/classification. Accessed January 15, 2019.

10. Neena IE, Poornima P, Edagunji G, Roopa KB, Bharath KP. Lasers in pediatric dentistry: A review. Int J Contemp Dent Med Rev. 2015; 2015:030115.

11. Das UK, Maiti N. Versatile diode. J Dent Med Sci. 2013;6(1):1-6. doi: 10.9790/0853-0610106.

12. Thornhill MH, Dayer M, Lockhart PB, McGurk M, Shanson D, Prendergast B, et al. Guidelines on prophylaxis to prevent infective endocarditis. Br Dent J. 2016;220(2):516. doi: 10.1038/sj.bdj.2016.49. 